\title{
Cardinal sequences and Cohen real extensions
}

\author{
by \\ István Juhász (Budapest), Saharon Shelah (Jerusalem), \\ Lajos Soukup (Budapest) and Zoltán Szentmiklóssy (Budapest)
}

\begin{abstract}
We show that if we add any number of Cohen reals to the ground model then, in the generic extension, a locally compact scattered space has at most $\left(2^{\aleph_{0}}\right)^{V}$ levels of size $\omega$. We also give a complete ZFC characterization of the cardinal sequences of regular scattered spaces. Although the classes of regular and of 0 -dimensional scattered spaces are different, we prove that they have the same cardinal sequences.
\end{abstract}

1. Introduction. Let us start by recalling that a topological space $X$ is called scattered if every non-empty subspace of $X$ has an isolated point. Via the well-known Cantor-Bendixson analysis then $X$ decomposes into levels; the $\alpha$ th Cantor-Bendixson level of $X$ will be denoted by $\mathrm{I}_{\alpha}(X)$. The height of $X, \operatorname{ht}(X)$, is the least ordinal $\alpha$ with $\mathrm{I}_{\alpha}(X)=\emptyset$. The width of $X, \operatorname{wd}(X)$, is defined by

$$
\operatorname{wd}(X)=\sup \left\{\left|\mathrm{I}_{\alpha}(X)\right|: \alpha<\operatorname{ht}(X)\right\} .
$$

Our main object of study is the cardinal sequence of $X$, denoted by $\operatorname{CS}(X)$, which is the sequence of cardinalities of the non-empty Cantor-Bendixson levels of $X$, i.e.

$$
\mathrm{CS}(X)=\left\langle\left|\mathrm{I}_{\alpha}(X)\right|: \alpha<\operatorname{ht}(X)\right\rangle .
$$

The cardinality of a $T_{3}$, in particular of a locally compact, scattered $T_{2}$ (in short: LCS ) space $X$ is at most $2^{\left|\mathrm{I}_{0}(X)\right|}$, hence clearly ht $(X)<\left(2^{\left|\mathrm{I}_{0}(X)\right|}\right)^{+}$and $\left|I_{\alpha}(X)\right| \leq 2^{\left|I_{0}(X)\right|}$ for each $\alpha$. (Locally compact scattered spaces are closely related to superatomic boolean algebras via Stone duality and the study of

2000 Mathematics Subject Classification: 54A25, 06E05, 54G12, 03E35.

Key words and phrases: locally compact scattered space, superatomic Boolean algebra, Cohen reals, cardinal sequence, regular space, 0-dimensional.

The first, third and fourth authors were supported by the Hungarian National Foundation for Scientific Research (OTKA) grant no. 37758.

The second author was supported by The Israel Science Foundation founded by the Israel Academy of Sciences and Humanities. Publication 765.

The third author was partially supported by Grant-in-Aid for JSPS Fellows No. 98259 of the Ministry of Education, Science, Sports and Culture, Japan. 
their cardinal sequences was actually originated within that subject.) Thus, in particular, under $\mathrm{CH}$ there is no scattered $T_{3}$ space of height $\omega_{2}$ and having only countably many isolated points. After I. Juhász and W. Weiss ([5, Theorem 4]) had proved in ZFC that for every $\alpha<\omega_{2}$ there is an LCS space $X$ with $\operatorname{ht}(X)=\alpha$ and $\operatorname{wd}(X)=\omega$, it was a natural question if the existence of an LCS space of height $\omega_{2}$ and width $\omega$ follows from $\neg \mathrm{CH}$. This question was answered in the negative by W. Just who proved ([6, Theorem 2.13]) that if one blows up the continuum by adding Cohen reals to a model of $\mathrm{CH}$ then in the resulting generic extension there is no LCS space of height $\omega_{2}$ and width $\omega$. On the other hand, in their ground breaking work [1], J. Baumgartner and S. Shelah produced a model in which there is an LCS space of height $\omega_{2}$ and width $\omega$, moreover they proved in ZFC that for each $\alpha<\left(2^{\omega}\right)^{+}$there is a scattered 0 -dimensional $T_{2}$ space $X$ with $\operatorname{ht}(X)=\alpha$ and $\operatorname{wd}(X)=\omega$.

Building on the idea of the proof of this latter result, in Section 3 we succeed in giving a complete characterization of the cardinal sequences of both $T_{3}$ and zero-dimensional $T_{2}$ scattered spaces. Although the classes of regular and of zero-dimensional scattered spaces are different, it will turn out that they yield the same class of cardinal sequences. We should add that, with quite a bit of extra effort, in [8], J. C. Martínez extended the former result of Baumgartner and Shelah by producing a model in which for every ordinal $\alpha<\omega_{3}$ there is an LCS space of height $\alpha$ and width $\omega$. The question if it is consistent to have an LCS space of height $\omega_{3}$ and width $\omega$ remains a big mystery.

In Section 2 we strengthen the result of Just by proving, in particular, that in the same Cohen real extension no LCS space may have $\omega_{2}$ countable (non-empty) levels. It seems to be an intriguing (and natural) problem whether the non-existence of an LCS space of width $\omega$ and height $\omega_{2}$ implies in ZFC the above conclusion, or more generally: when is a subsequence of the cardinal sequence of an LCS space again such a cardinal sequence? In connection with this problem let us remark that (as shown in [2] or [3]), in the side-by-side random real extension of a model of $\mathrm{CH}$ the combinatorial principle $\mathcal{C}^{s}\left(\omega_{2}\right)$ introduced in [4, Definition 2.3] holds, and consequently in such an extension there is no LCS space $X$ of height $\omega_{2}$ and width $\omega$. In fact, by [4, Theorem 4.12], $\mathcal{C}^{s}\left(\omega_{2}\right)$ implies that $\left\{\alpha \in \omega_{2}:\left|\mathrm{I}_{\alpha}(X)\right|=\omega\right\}$ is non-stationary in $\omega_{2}$. However, we do not know if our above mentioned result, namely Theorem 2.1 , is implied by $\mathcal{C}^{s}\left(\omega_{2}\right)$.

The moral of our above discussion may be concisely formulated as follows: The cardinal sequences of regular or zero-dimensional scattered spaces are only subject to the trivial inequality $|X| \leq 2^{\left|I_{0}(X)\right|}$, however those of the LCS spaces are much harder to determine, in particular, they are sensitive to the model of set theory in which we look at them. 
2. Countable levels in Cohen real extensions. Let us now formulate the promised strengthening of Just's result. We note that no assumption (such as $\mathrm{CH}$ ) is made on our ground model.

TheOREM 2.1. Set $\kappa=\left(2^{\omega}\right)^{+}$and add any number of Cohen reals to our ground model. Then in the resulting extension no LCS space contains a $\kappa$-sequence $\left\{E_{\alpha}: \alpha<\kappa\right\}$ of pairwise disjoint countable subspaces such that $\bar{E}_{\alpha} \supset E_{\beta}$ for all $\alpha<\beta<\kappa$. In particular, for any LCS space $X$ we have $\left|\left\{\alpha:\left|\mathrm{I}_{\alpha}(X)\right|=\omega\right\}\right|<\kappa$.

In fact, we shall prove a more general statement, but to formulate it we need a definition. A family $\mathcal{D}=\left\{\left\langle D_{0}^{\alpha}, D_{1}^{\alpha}\right\rangle: \alpha \in I\right\}$ of pairs (of sets) is said to be dyadic over a set $T$ if $D_{0}^{\alpha} \cap D_{1}^{\alpha}=\emptyset$ for each $\alpha \in I$ and

$$
\mathcal{D}[\varepsilon]=\bigcap\left\{D_{\varepsilon(\alpha)}^{\alpha}: \alpha \in \operatorname{dom} \varepsilon\right\}
$$

intersects $T$ for each $\varepsilon \in \operatorname{Fn}(I, 2)$. We simply say that $\mathcal{D}$ is dyadic if it is dyadic over some $T$, i.e. $\mathcal{D}[\varepsilon] \neq \emptyset$ for each $\varepsilon \in \operatorname{Fn}(I, 2)$. (As usual, $\operatorname{Fn}(I, 2)$ denotes the set of all finite partial functions from $I$ into 2.)

Now, it is obvious that in an LCS space

- the compact open sets form a base that is closed under finite unions,

- there is no infinite dyadic system of pairs of compact sets.

Consequently, Theorem 2.2 below immediately yields Theorem 2.1 above.

TheOREm 2.2. Set $\kappa=\left(2^{\omega}\right)^{+}$and add any number of Cohen reals to the ground model. Then in the resulting generic extension the following statement holds: If $X$ is any $T_{2}$ space containing pairwise disjoint countable subspaces $\left\{E_{\alpha}: \alpha<\kappa\right\}$ such that $\bar{E}_{\alpha} \supset E_{\beta}$ for $\alpha<\beta<\kappa$ and $X=\bar{E}_{0}$ (i.e. $E_{0}$ is dense in $X$ ), and moreover, for each $x \in X$, we have fixed a neighbourhood base $\mathcal{B}(x)$ of $x$ in $X$ that is closed under finite unions then there is an infinite set $a \in[\kappa]^{\omega}$, for each $\alpha \in a$ there are disjoint finite subsets $L_{\alpha}^{0}$ and $L_{\alpha}^{1}$ of $E_{\alpha}$, and for each $x \in L_{\alpha}^{0} \cup L_{\alpha}^{1}$ there is a basic neighbourhood $V(x) \in \mathcal{B}(x)$ such that the infinite family of pairs

$$
\left\{\left\langle\bigcup_{x \in L_{\alpha}^{0}} V(x), \bigcup_{x \in L_{\alpha}^{1}} V(x)\right\rangle: \alpha \in a\right\}
$$

is dyadic.

This topological statement in the Cohen extension in turn will follow from a purely combinatorial one concerning certain matrices, namely Theorem 2.7.

To formulate this theorem we again need some notation and definitions.

For an ordinal $\alpha$ the interval $[\omega \alpha, \omega \alpha+\omega)$ will be denoted by $\mathbb{I}_{\alpha}$.

Given two sets $A$ and $B$ we write $f: A \stackrel{\mathrm{p}}{\rightarrow} B$ to denote that $f$ is a partial function from $A$ to $B$, i.e. a function from a subset of $A$ into $B$. As usual, 
we let

$$
\operatorname{Fn}(A, B)=\{f:|f|<\omega \text { and } f: A \stackrel{\mathrm{p}}{\rightarrow} B\} .
$$

If $A \subset$ On then for any partial function $f: A \stackrel{\mathrm{p}}{\rightarrow} B$ we set

We let

$$
\gamma(f)= \begin{cases}\min \operatorname{dom} f & \text { if } \operatorname{dom} f \neq \emptyset, \\ \sup A & \text { if } \operatorname{dom} f=\emptyset .\end{cases}
$$

$$
\Omega=\left\{\langle A, B\rangle \in[\omega]^{<\omega} \times[\omega]^{<\omega}: A \cap B=\emptyset\right\},
$$

and for $\ell=\langle A, B\rangle \in \Omega$ we set $\pi_{0}(\ell)=A$ and $\pi_{1}(\ell)=B$.

If $S$ and $T$ are sets of ordinals, we denote by $\mathcal{M}(S, T)$ the family of all $S \times \omega$-matrices consisting of subsets of $T$, i.e. $\mathcal{A} \in \mathcal{M}(S, T)$ means that $\mathcal{A}=\left\langle A_{\alpha, i}: \alpha \in S, i \in \omega\right\rangle$, where $A_{\alpha, i} \subset T$ for each $\alpha \in S$ and $i<\omega$.

For $\mathcal{A} \in \mathcal{M}(S, T), f: S \stackrel{\mathrm{p}}{\rightarrow} S$, and $s: S \stackrel{\mathrm{p}}{\rightarrow} \Omega$ the pair $(f, s)$ is said to be $\mathcal{A}$-dyadic (over $U$ ) if the family of pairs

$$
\begin{aligned}
\left\{\left\langle\bigcup\left\{A_{f(\alpha), n}: n \in \pi_{0}(s(\alpha))\right\}, \bigcup\left\{A_{f(\alpha), n}: n \in \pi_{1}(s(\alpha))\right\}\right\rangle:\right. & \\
\alpha \in \operatorname{dom} f & \cap \operatorname{dom} s\}
\end{aligned}
$$

is dyadic (over $U$ ). If the pair $\left\langle\operatorname{id}_{S}, s\right\rangle$ is $\mathcal{A}$-dyadic (over $U$ ) then $s$ is simply called $\mathcal{A}$-dyadic (over $U$ ). It is this latter notion of $\mathcal{A}$-dyadicity of a single partial function that is really important (that for pairs is only of technical significance). Hence we state below an alternative characterization of it.

For $\mathcal{A} \in \mathcal{M}(S, T), s: S \stackrel{\mathrm{p}}{\rightarrow} \Omega$, and $\varepsilon \in \mathrm{Fn}(\operatorname{dom} s, 2)$ we write

$$
\mathcal{A}[s, \varepsilon]=\bigcap_{\alpha \in \operatorname{dom} \varepsilon} \bigcup\left\{A_{\alpha, n}: n \in \pi_{\varepsilon(\alpha)}(s(\alpha))\right\} .
$$

Observation 2.3. If $\mathcal{A} \in \mathcal{M}(S, T)$ then $s: S \stackrel{\mathrm{p}}{\rightarrow} \Omega$ is $\mathcal{A}$-dyadic over $U$ iff $\mathcal{A}[s, \varepsilon] \cap U \neq \emptyset$ for each $\varepsilon \in \operatorname{Fn}(\operatorname{dom} s, 2)$ and

$$
\bigcup\left\{A_{\alpha, n}: n \in \pi_{0}(s(\alpha))\right\} \cap \bigcup\left\{A_{\alpha, n}: n \in \pi_{1}(s(\alpha))\right\}=\emptyset
$$

for each $\alpha \in \operatorname{dom} s$.

The following easy observation will be applied later, in the proof of Lemma 2.9:

Observation 2.4. If $g: S \stackrel{\mathrm{p}}{\rightarrow} S$ and $s: S \stackrel{\mathrm{p}}{\rightarrow} \Omega$ satisfy dom $s \subset \operatorname{ran} g$, and the pair $(g, s \circ g)$ is $\mathcal{A}$-dyadic over $U$, then $s$ is $\mathcal{A}$-dyadic over $U$ as well.

Definition 2.5. Fix a cardinal $\kappa$ and let $\mathcal{D} \in \mathcal{M}(\kappa, \kappa)$. For $s: \kappa \stackrel{\mathrm{p}}{\rightarrow} \Omega$ we say that $s$ is $\mathcal{D}$-min-dyadic (m.d.) if $s$ is $\mathcal{D}$-dyadic over $\mathbb{I}_{\gamma(s)}$.

Moreover, we say that the matrix $\mathcal{D}$ is m.d.-extendible if for each finite $\mathcal{D}$-min-dyadic partial function $s: \kappa \stackrel{\mathrm{p}}{\rightarrow} \Omega$ and for each $\gamma<\gamma(s)$ there is an $\ell \in \Omega$ such that $s \cup\{\langle\gamma, \ell\rangle\}$ is also $\mathcal{D}$-min-dyadic, i.e. $\mathcal{D}$-dyadic over $\mathbb{I}_{\gamma}$. 
Since $\mathbb{I}_{0}=\omega$, we clearly have the following.

OBSERVATION 2.6. If $\mathcal{D} \in \mathcal{M}(\kappa, \kappa)$ is m.d-extendible and $s: \kappa \stackrel{\mathrm{p}}{\rightarrow} \Omega$ is a finite $\mathcal{D}$-min-dyadic partial function then $s$ is $\mathcal{D}$-dyadic over $\omega$.

Finally, a matrix $\mathcal{D} \in \mathcal{M}(\kappa, \kappa)$ will be called $\omega$-determined if $D_{\alpha, n} \cap$ $D_{\alpha, m} \cap \omega=\emptyset$ implies $D_{\alpha, n} \cap D_{\alpha, m}=\emptyset$ whenever $\alpha<\kappa$ and $n<m<\omega$.

We now have all the necessary ingredients to formulate and prove the promised combinatorial statement that will be valid in any Cohen real extension.

TheOREM 2.7. Set $\kappa=\left(2^{\omega}\right)^{+}$and add any number of Cohen reals to the ground model. Then in the resulting generic extension for every $\omega$ determined and m.d.-extendible matrix $\mathcal{D} \in \mathcal{M}(\kappa, \kappa)$ there is an infinite $\mathcal{D}$-dyadic partial function $h: \kappa \stackrel{\mathrm{p}}{\rightarrow} \Omega$.

Before proving Theorem 2.7, however, we show how Theorem 2.2 can be deduced from it.

Proof of Theorem 2.2 using Theorem 2.7. We can assume without any loss of generality that $E_{\alpha}=\mathbb{I}_{\alpha}$ for each $\alpha<\kappa$. We will define an appropriate matrix $\mathcal{D} \in \mathcal{M}(\kappa, \kappa)$.

To this end, for coding purposes, we first fix a bijection $\varrho:[\omega]^{2} \rightarrow \omega$ and let $\eta: \omega \rightarrow \omega$ and $\nu: \omega \rightarrow \omega$ be the "co-ordinate" functions of its inverse, i.e. $k=\varrho(\{\nu(k), \eta(k)\})$ and $\nu(k)<\eta(k)$ for each $k<\omega$.

Since $X$ is $T_{2}$, for each $n<\omega$ we can simultaneously pick basic neighbourhoods $B_{n}^{\alpha}(m) \in \mathcal{B}(\omega \alpha+m)$ of the points $\omega \cdot \alpha+m \in E_{\alpha}=\mathbb{I}_{\alpha}$ for all $m<n$ such that the sets $\left\{B_{n}^{\alpha}(m): m<n\right\}$ are pairwise disjoint.

Now we define $\mathcal{D}=\left\langle D_{\alpha, k}:\langle\alpha, k\rangle \in \kappa \times \omega\right\rangle \in \mathcal{M}(\kappa, \kappa)$ as follows:

$$
D_{\alpha, k}=B_{\eta(k)}^{\alpha}(\nu(k)) \cap \kappa .
$$

This matrix $\mathcal{D}$ is clearly $\omega$-determined because $E_{0}=\mathbb{I}_{0}=\omega$ is dense in $X$. It is a bit less easy to establish the following

Claim. $\mathcal{D}$ is also m.d.-extendible.

Proof. Let $s: \kappa \stackrel{\mathrm{p}}{\rightarrow} \Omega$ be a finite $\mathcal{D}$-min-dyadic partial function and let $\gamma<\gamma(s)$.

Since the sets $\left\{\mathcal{D}[s, \varepsilon]: \varepsilon \in \operatorname{dom}^{s} 2\right\}$ are all open in the subspace $\kappa$ and they all intersect $\mathbb{I}_{\gamma(s)}$, and moreover every element of $\mathbb{I}_{\gamma(s)}$ is an accumulation point of $\mathbb{I}_{\gamma}$, it follows that $\mathcal{D}[s, \varepsilon] \cap \mathbb{I}_{\gamma}$ must be infinite for each $\varepsilon \in \operatorname{dom} s 2$. Thus we can easily pick two disjoint finite subsets $A_{0}$ and $A_{1}$ of $\mathbb{I}_{\gamma}$ such that every $\mathcal{D}[s, \varepsilon]$ intersects both $A_{0}$ and $A_{1}$. Let $n<\omega$ be chosen in such a way that $A_{0} \cup A_{1} \subset\{\omega \gamma+m: m<n\}$, and set $K_{i}=\left\{\varrho\{m, n\}: m<n \wedge \omega \gamma+m \in A_{i}\right\}$ for $i<2$. Since $\varrho$ is one-to-one we 
have $K_{0} \cap K_{1}=\emptyset$, hence $\ell=\left\langle K_{0}, K_{1}\right\rangle \in \Omega$, and moreover

$$
\left(\bigcup_{m \in K_{0}} D_{\gamma, m}\right) \cap\left(\bigcup_{m \in K_{1}} D_{\gamma, m}\right)=\emptyset
$$

because the elements of the family $\left\{B_{n}^{\gamma}(m): m<n\right\}$ are pairwise disjoint.

Now put $t=s \cup\{\langle\gamma, \ell\rangle\}$. Then for each $\varepsilon \in{ }^{\operatorname{dom} t} 2$ we clearly have

$$
A_{\varepsilon(\gamma)} \cap \mathcal{D}[t, \varepsilon] \neq \emptyset,
$$

hence $(\star)$ and $(\star \star)$ together imply that the extension $t$ of $s$ is $\mathcal{D}$-dyadic over $\mathbb{I}_{\gamma}=\mathbb{I}_{\gamma(t)} \cdot \mathbf{m}_{\text {Claim }}$

Thus we may apply Theorem 2.7 to the matrix $\mathcal{D}$ to obtain an infinite $\mathcal{D}$-dyadic partial function $h: \kappa \stackrel{\mathrm{p}}{\rightarrow} \Omega$. Set $a=\operatorname{dom} h$ and for each $\alpha \in a$ and $i<2$ put $L_{\alpha}^{i}=\left\{\omega \alpha+\nu(k): k \in \pi_{i}(h(\alpha))\right\}$. For $x \in L_{\alpha}^{i}$ put

$$
V(x)=\bigcup\left\{B_{\eta(k)}^{\alpha}(\nu(k)): x=\omega \alpha+\nu(k) \text { and } k \in \pi_{i}(h(\alpha))\right\} .
$$

Then $V(x) \in \mathcal{B}(x)$ because $\mathcal{B}(x)$ is closed under finite unions. Since for $i<2$,

$$
\left(\bigcup\left\{V(x): x \in L_{\alpha}^{i}\right\}\right) \cap \kappa=\bigcup\left\{D_{\alpha, k}: k \in \pi_{i}(h(\alpha))\right\}
$$

and

$$
\bigcup\left\{D_{\alpha, k}: k \in \pi_{0}(h(\alpha))\right\} \cap \bigcup\left\{D_{\alpha, k}: k \in \pi_{1}(h(\alpha))\right\}=\emptyset,
$$

we have

$$
\left(\bigcup\left\{V(x): x \in L_{\alpha}^{0}\right\}\right) \cap\left(\bigcup\left\{V(x): x \in L_{\alpha}^{1}\right\}\right)=\emptyset
$$

because the latter intersection is an open set which does not intersect the dense set $\mathbb{I}_{0} \subset \kappa$. Hence the infinite family

$$
\left\{\left\langle\bigcup_{x \in L_{\alpha}^{0}} V(x), \bigcup_{x \in L_{\alpha}^{1}} V(x)\right\rangle: \alpha \in a\right\}
$$

is indeed dyadic. -2.2

Proof of Theorem 2.7. The proof will be based on the following two lemmas, 2.9 and 2.10. For these we need some more notation and a new and rather technical notion of extendibility for set matrices.

Given a set $A$ we define

$$
\mathcal{F}(A)=\{f \in \operatorname{Fn}(A, A): f \text { is injective and } \operatorname{dom}(f) \cap \operatorname{ran}(f)=\emptyset\} .
$$

Each function $f \in \mathcal{F}(A)$ can be extended in a natural way to a bijection $f^{*}: A \rightarrow A$ as follows:

$$
f^{*}(a)= \begin{cases}f(a) & \text { if } a \in \operatorname{dom} f, \\ f^{-1}(a) & \text { if } a \in \operatorname{ran} f, \\ a & \text { otherwise. }\end{cases}
$$


Definition 2.8. If $S$ and $T$ are sets of ordinals then the matrix $\mathcal{A} \in$ $\mathcal{M}(S, T)$ is called nicely extendible if for each $f \in \mathcal{F}(S)$ there are a family $N(f) \subset \operatorname{Fn}(S, \Omega)$ and a function $K^{f}: N(f) \rightarrow[S] \leq \omega$ such that

(1) the pair $(f, s)$ is $\mathcal{A}$-dyadic whenever $f \in \mathcal{F}(S)$ and $s \in N(f)$,

(2) $\emptyset \in N(f)$ for each $f \in \mathcal{F}(S)$,

(3) for $f, g \in \mathcal{F}(S)$ and $s \in N(f)$ if $f^{*}\left|K^{f}(s)=g^{*}\right| K^{f}(s)$ then $s \in$ $N(g)$.

(4) for any $f \in \mathcal{F}(S), s \in N(f)$ and $\alpha \in S \cap \gamma(s)$ there is $\ell \in \Omega$ such that $s \cup\{\langle\alpha, \ell\rangle\} \in N(f)$.

Clearly, this last condition (4) is what explains our terminology.

Lemma 2.9. If $\kappa>\omega_{1}$ is regular and $\mathcal{A} \in \mathcal{M}(\kappa, \omega)$ is a nicely extendible matrix then there is an infinite partial function $h: \kappa \stackrel{\mathrm{p}}{\rightarrow} \omega$ that is $\mathcal{A}$-dyadic.

Proof. By induction on $n \in \omega$ we will define functions $h_{0} \subset h_{1} \subset \cdots \subset$ $h_{n} \subset \cdots$ from $\operatorname{Fn}(\kappa, \Omega)$ such that $\left|h_{n}\right|=n$ and for each $\nu \in \kappa$,

$(*)_{\nu}^{n} \quad$ there is $g \in \mathcal{F}(\kappa)$ such that $\gamma(g)>\nu, \operatorname{ran} g=\operatorname{dom} h_{n}$ and $h_{n} \circ g$ $\in N(g)$.

First observe that $h_{0}=\emptyset$ satisfies our requirements because, according to $(2)$, condition $(*)_{\nu}^{0}$ holds trivially for each $\nu<\kappa$.

Next assume that the construction has been done and the induction hypothesis has been established for $n$. For each $\nu<\kappa$ choose a function $g_{\nu} \in \mathcal{F}(\kappa)$ witnessing $(*)_{\nu+\omega_{1}}^{n}$ and then write $K_{\nu}=K^{g_{\nu}}\left(h_{n} \circ g_{\nu}\right)$ and pick $\zeta_{\nu} \in\left(\nu, \nu+\omega_{1}\right) \backslash K_{\nu}$. Clearly the set

$$
L=\left\{\xi \in \kappa:\left|\left\{\nu<\kappa: \xi \notin K_{\nu}\right\}\right|<\kappa\right\}
$$

is countable and so we can pick $\xi_{n} \in \kappa \backslash\left(L \cup \operatorname{dom} h_{n}\right)$; then the set

$$
J=\left\{\nu<\kappa: \xi_{n} \notin K_{\nu}\right\}
$$

is of size $\kappa$.

Now set $g_{\nu}^{\prime}=g_{\nu} \cup\left\{\left\langle\zeta_{\nu}, \xi_{n}\right\rangle\right\}$ for every $\nu \in J$. For every such $\nu$ then $\zeta_{\nu}, \xi_{n} \notin K_{\nu}$ implies $g_{\nu}{ }^{*}\left\lceil K_{\nu}=g_{\nu}^{\prime *} \mid K_{\nu}\right.$, hence $h_{n} \circ g_{\nu} \in N\left(g_{\nu}^{\prime}\right)$ by (3). Since $\zeta_{\nu}<\nu+\omega_{1}<\gamma\left(g_{\nu}\right)=\gamma\left(h_{n} \circ g_{\nu}\right)$, we can now apply (4) to get $\ell^{\nu} \in \Omega$ such that $\left(h_{n} \circ g_{\nu}\right) \cup\left\{\left\langle\zeta_{\nu}, \ell^{\nu}\right\rangle\right\} \in N\left(g_{\nu}^{\prime}\right)$.

We can now fix $\ell_{n} \in \Omega$ such that $J_{n}=\left\{\nu \in J: \ell^{\nu}=\ell_{n}\right\}$ is of size $\kappa$ and let $h_{n+1}=h_{n} \cup\left\{\left\langle\xi_{n}, \ell_{n}\right\rangle\right\}$.

If $\nu \in J_{n}$ then $h_{n+1} \circ g_{\nu}^{\prime}=\left(h_{n} \circ g_{\nu}\right) \cup\left\{\left\langle\zeta_{\nu}, \ell_{n}\right\rangle\right\} \in N\left(g_{\nu}^{\prime}\right)$ and $\gamma\left(g_{\nu}^{\prime}\right)>\nu$, so $g_{\nu}^{\prime}$ witnesses $(*)_{\nu}^{n+1}$. But $J_{n}$ is unbounded in $\kappa$, hence the inductive step is completed.

By $(*)_{0}^{n}$, for each $n<\omega$ there is $g_{n}$ such that $\operatorname{dom} h_{n}=\operatorname{ran} g_{n}$ and $h_{n} \circ g_{n} \in N\left(g_{n}\right)$. Hence, by $(1),\left(g_{n}, h_{n} \circ g_{n}\right)$ is $\mathcal{A}$-dyadic, and so $h_{n}$ is 
$\mathcal{A}$-dyadic according to Observation 2.4. Consequently, $h=\bigcup\left\{h_{n}: n<\omega\right\}$ is as required: it is $\mathcal{A}$-dyadic and infinite. 2.9

Given any infinite set $I$ we denote by $\mathcal{C}_{I}$ the poset $\operatorname{Fn}(I, 2)$, i.e. the standard notion of forcing that adds $|I|$ Cohen reals.

Lemma 2.10. Let $\kappa=\left(2^{\omega}\right)^{+}$. Then for each $\lambda$ we have $V^{\mathcal{C}_{\lambda}}=$ "If $\mathcal{D} \in \mathcal{M}(\kappa, \kappa)$ is both $\omega$-determined and m.d.-extendible then there is $I \in[\kappa]^{\kappa}$ such that $\mathcal{D}^{*}=\left\langle D_{\alpha, n} \cap \omega:\langle\alpha, n\rangle \in I \times \omega\right\rangle$ is nicely extendible."

Proof. Assume that

$$
1_{\mathcal{C}_{\lambda}} \Vdash \dot{\mathcal{D}} \in \mathcal{M}(\kappa, \kappa) \text { is m.d.-extendible. }
$$

Let $\theta$ be a large enough regular cardinal and consider the structure $\mathcal{H}_{\theta}=$ $\left\langle H_{\theta}, \in, \triangleleft, \kappa, \lambda, \dot{\mathcal{D}}\right\rangle$, where $H_{\theta}=\{x:|\mathrm{TC}(x)|<\theta\}$ and $\triangleleft$ is a fixed wellordering of $H_{\theta}$.

Working in $V$, for each $\alpha<\kappa$ choose a countable elementary submodel $N_{\alpha}$ of $\mathcal{H}_{\theta}$ with $\alpha \in N_{\alpha}$. Then there is $I \in[\kappa]^{\kappa}$ such that the models $\left\{N_{\alpha}\right.$ : $\alpha \in I\}$ are not only pairwise isomorphic but, denoting by $\sigma_{\alpha, \beta}$ the unique isomorphism between $N_{\alpha}$ and $N_{\beta}$, we have

(i) the family $\left\{N_{\alpha} \cap \theta: \alpha \in I\right\}$ forms a $\Delta$-system with kernel $\Lambda$,

(ii) $\sigma_{\alpha, \beta}(\xi)=\xi$ for each $\xi \in \Lambda$,

(iii) $\sigma_{\alpha, \beta}(\alpha)=\beta$.

For each $\alpha<\kappa$ and $n<\omega$ let $\dot{D}_{\alpha, n}$ be the $\triangleleft$-minimal $\mathcal{C}_{\lambda}$-name of the $\langle\alpha, n\rangle$ th entry of $\dot{\mathcal{D}}$. Since $\triangleleft$ is in $\mathcal{H}_{\theta}$ and $\sigma_{\alpha, \beta}(\alpha)=\beta$ we have

Claim 2.10.1. $\sigma_{\alpha, \beta}\left(\dot{D}_{\alpha, n}\right)=\dot{D}_{\beta, n}$ for all $\alpha, \beta \in I$ and $n \in \omega$.

Let $G$ be any $\mathcal{C}_{\lambda}$-generic filter over $V$. We shall show that

$$
V[G] \models \text { "D } \mathcal{D}^{*}=\left\langle D_{\alpha, n} \cap \omega:\langle\alpha, n\rangle \in I \times \omega\right\rangle \text { is nicely extendible." }
$$

For each $f \in \mathcal{F}(I)$ define the bijection $\varrho_{f}: \lambda \rightarrow \lambda$ as follows:

$$
\varrho_{f}(\xi)= \begin{cases}\sigma_{\alpha, f^{*}(\alpha)}(\xi) & \text { if } \xi \in N_{\alpha} \cap \lambda \text { for some } \alpha \in I, \\ \xi & \text { otherwise. }\end{cases}
$$

In a natural way $\varrho_{f}$ extends to an automorphism of $\mathcal{C}_{\lambda}$, which will be denoted by $\varrho_{f}$ as well. Clearly, we have

Claim 2.10.2. If $f \in \mathcal{F}(I), f(\alpha)=\beta, p \in \mathcal{C}_{\lambda} \cap N_{\alpha}$, then $\sigma_{\alpha, \beta}(p)=\varrho_{f}(p)$.

For $f \in \mathcal{F}(I)$ let $G^{f}=\left\{\varrho_{f}^{-1}(p): p \in G\right\}$ and then set

$$
\begin{aligned}
N(f) & =\left\{s \in \operatorname{Fn}(I, \Omega): s \text { is } \dot{\mathcal{D}}\left[G^{f}\right] \text {-min-dyadic }\right\} \\
& =\left\{s \in \operatorname{Fn}(I, \Omega): \exists q \in G^{f} q \Vdash \text { "s is } \dot{\mathcal{D}} \text {-min-dyadic" }\right\} .
\end{aligned}
$$


To define $K^{f}$, for each $s \in N(f)$ pick a condition $p_{s} \in G$ such that

$$
\varrho_{f}^{-1}\left(p_{s}\right) \Vdash s \text { is } \dot{\mathcal{D}} \text {-min-dyadic }
$$

and let

$$
K^{f}(s)=\left\{\alpha \in I:\left(N_{\alpha} \backslash \Lambda\right) \cap \operatorname{dom} p_{s} \neq \emptyset\right\} .
$$

Note that $K^{f}(s)$ as defined above is finite, although 2.8(3) only requires $K^{f}(s)$ to be countable.

To check property 2.8(3) assume that $f, g \in \mathcal{F}(I)$ and $s \in N(f)$ with $g^{*}\left\lceil K^{f}(s)=f^{*} \mid K^{f}(s)\right.$. Then $\varrho_{g}^{-1}\left(p_{s}\right)=\varrho_{f}^{-1}\left(p_{s}\right)$ and so

$$
\varrho_{g}^{-1}\left(p_{s}\right) \Vdash s \text { is } \dot{\mathcal{D}} \text {-min-dyadic, }
$$

hence $s$ is also $\dot{\mathcal{D}}\left[G^{g}\right]$-min-dyadic, i.e. $s \in N(g)$.

Before checking 2.8(1) we need one more observation.

Claim 2.10.3. $\dot{D}_{f(\alpha), n}[G] \cap \omega=\dot{D}_{\alpha, n}\left[G^{f}\right] \cap \omega$ whenever $f \in \mathcal{F}(I), \alpha \in$ $\operatorname{dom} f$, and $n<\omega$.

Proof. Let $k \in \omega$. Then $k \in \dot{D}_{f(\alpha), n}[G]$ iff $\exists p \in G p \Vdash " k \in \dot{D}_{f(\alpha), n}$ " iff $\exists p \in G \cap N_{f(\alpha)} p \Vdash " k \in \dot{D}_{f(\alpha), n}$ " iff $\exists q \in G^{f} \cap N_{\alpha} p=\sigma_{\alpha, f(\alpha)}(q) \Vdash$ " $k \in \dot{D}_{f(\alpha), n}$ " iff $\exists q \in G^{f} \cap N_{\alpha} q \Vdash$ " $k \in \dot{D}_{\alpha, n}$ " iff $\exists q \in G^{f} q \Vdash " k \in \dot{D}_{\alpha, n}$ " iff $k \in \dot{D}_{\alpha, n}\left[G^{f}\right] \cdot \mathbf{-}_{2.10}$

Now let $f \in \mathcal{F}(I)$ and $s \in N(f)$. By the definition of $N(f), s$ is $\dot{\mathcal{D}}\left[G^{f}\right]$ min-dyadic and so by Observation 2.6, s is $\dot{\mathcal{D}}\left[G^{f}\right]$-dyadic over $\omega$. But it follows from 2.10 .3 that $s$ is $\dot{\mathcal{D}}\left[G^{f}\right]$-dyadic over $\omega$ if and only if the pair $(f, s)$ is $\dot{\mathcal{D}}[G]$-dyadic over $\omega$.

$2.8(2)$ is clear because $\emptyset$ is trivially $\mathcal{A}$-min-dyadic for any $\mathcal{A} \in \mathcal{M}(\kappa, \omega)$. Finally, 2.8(4) follows from the definition of $N(f)$ because $\dot{\mathcal{D}}\left[G^{f}\right]$ is m.d.extendible. 2.10

Now, to complete the proof of Theorem 2.7, first apply Lemma 2.10 to get $I \in[\kappa]^{\kappa}$ such that

$$
\mathcal{D}^{*}=\left\langle D_{\alpha, n} \cap \omega:\langle\alpha, n\rangle \in I \times \omega\right\rangle
$$

is nicely extendible. Then applying Lemma 2.9 to $\mathcal{D}^{*}$ we obtain an infinite $\mathcal{D}^{*}$-dyadic function $h: \kappa \stackrel{\mathrm{p}}{\rightarrow} \Omega$. Since the matrix $\mathcal{D}$ is $\omega$-determined the function $h$ is $\mathcal{D}$-dyadic as well.

3. Cardinal sequences of regular and 0-dimensional spaces. For convenience, in this section, space will always mean Hausdorff space. Then for any regular, scattered space $X$ we have $|X| \leq 2^{\left|I_{0}(X)\right|}$, hence for such a space $X$ its cardinal sequence $s$ satisfies length $(s)<\left(2^{\left|\mathrm{I}_{0}(X)\right|}\right)^{+}$and $s(\alpha) \leq$ $2^{s(\beta)}$ whenever $\beta<\alpha$. We shall show below that these properties of a sequence $s$ actually characterize the cardinal sequences of regular scattered spaces. 
In [1], for each $\gamma<\left(2^{\omega}\right)^{+}$, a 0-dimensional, scattered space of height $\gamma$ and width $\omega$ was constructed. The next lemma generalizes that construction.

For an infinite cardinal $\kappa$, let $S_{\kappa}$ be the following family of sequences of cardinals:

$S_{\kappa}=\left\{\left\langle\kappa_{\alpha}: \alpha<\delta\right\rangle: \delta<\left(2^{\kappa}\right)^{+}, \kappa_{0}=\kappa\right.$ and $\kappa \leq \kappa_{\alpha} \leq 2^{\kappa}$ for each $\left.\alpha<\delta\right\}$.

Lemma 3.1. For any infinite cardinal $\kappa$ and $s \in S_{\kappa}$ there is a 0-dimensional scattered space $X$ with $\operatorname{CS}(X)=s$.

Proof. Let $s=\left\langle\kappa_{\alpha}: \alpha<\delta\right\rangle \in S_{\kappa}$. Write $X=\bigcup\left\{\{\alpha\} \times \kappa_{\alpha}: \alpha<\delta\right\}$. Since $|X| \leq 2^{\kappa}$ we can fix an independent family $\left\{F_{x}: x \in X\right\} \subset[\kappa]^{\kappa}$.

The underlying set of our space is $X$ and the topology $\tau$ on $X$ is given by declaring for each $x=\langle\alpha, \xi\rangle \in X$ the set

$$
U_{x}=\{x\} \cup\left(\alpha \times F_{x}\right)
$$

to be clopen, i.e. $\left\{U_{x}, X \backslash U_{x}: x \in X\right\}$ is a subbase for $\tau$.

The space $X$ is clearly 0 -dimensional and $T_{2}$.

Claim 3.1.1. If $x=\langle\beta, \xi\rangle \in U \in \tau$ and $\alpha<\beta$ then $U \cap\left(\{\alpha\} \times \kappa_{\alpha}\right)$ is infinite.

Proof. We can find disjoint sets $A, B \in[X \backslash\{x\}]^{<\omega}$ such that

$$
x \in U_{x} \cap \bigcap_{y \in A} U_{y} \backslash \bigcup_{z \in B} U_{z} \subset U .
$$

Observe that if $\langle\gamma, \xi\rangle \in A$ then $\beta<\gamma$. Thus

$$
U \cap\left(\{\alpha\} \times \kappa_{\alpha}\right) \supset\{\alpha\} \times\left(\bigcap_{y \in A \cup\{x\}} F_{y} \backslash \bigcup_{z \in B} F_{z}\right),
$$

and the set on the right side is infinite because $\left\{F_{x}: x \in X\right\}$ was chosen to be independent.

To complete the proof of 3.1 , by induction on $\alpha<\kappa$, we verify that $\mathrm{I}_{\alpha}(X)=\{\alpha\} \times \kappa_{\alpha}$, hence $\operatorname{CS}(X)=s$. Assume that this is true for $\nu<\alpha$. If $x \in\{\alpha\} \times \kappa_{\alpha}$ then

$$
U_{x} \cap\left(X \backslash \bigcup_{\nu<\alpha} \mathrm{I}_{\nu}(X)\right)=\{x\},
$$

hence $\{\alpha\} \times \kappa_{\alpha} \subset \mathrm{I}_{\alpha}(X)$. On the other hand, if $x=\langle\beta, \xi\rangle \in X$ with $\beta>\alpha$ and $U \in \tau$ is a neighbourhood of $x$, then, by the claim above, $U \cap\left(\{\alpha\} \times \kappa_{\alpha}\right)$ is infinite, hence $x$ is not isolated in $X \backslash \bigcup_{\nu<\alpha} \mathrm{I}_{\nu}(X)$, i.e., $x \notin \mathrm{I}_{\alpha}(X)$. Thus $\mathrm{I}_{\alpha}(X)=\{\alpha\} \times \kappa_{\alpha} \cdot \mathbf{-} 3.1$

THEOREM 3.2. For any sequence $s$ of cardinals the following statements are equivalent:

(1) $s=\mathrm{CS}(X)$ for some regular scattered space $X$,

(2) $s=\mathrm{CS}(X)$ for some 0 -dimensional scattered space $X$, 
(3) for some natural number $m$ there are infinite cardinals $\kappa_{0}>\kappa_{1}>$ $\cdots>\kappa_{m-1}$ and for all $i<m$ sequences $s_{i} \in S_{\kappa_{i}}$ such that $s=$ $s_{0} \frown s_{1} \frown \ldots \frown s_{m-1}$ or $s=s_{0} \frown s_{1} \frown \ldots \frown s_{m-1} \frown\langle n\rangle$ for some natural number $n>0$.

Proof. $(1) \Rightarrow(3)$. By induction on $j$ we choose ordinals $\nu_{j}<\mathrm{ht}(X)$ and cardinals $\kappa_{j}$ such that $\nu_{0}=0$ and $\kappa_{0}=\left|\mathrm{I}_{0}(X)\right|$, and moreover, for $j>0$ with $\kappa_{j-1}$ infinite,

$$
\nu_{j}=\min \left\{\nu \leq \mathrm{ht}(X):\left|\mathrm{I}_{\nu}(X)\right|<\kappa_{j-1}\right\},
$$

and $\kappa_{j}=\left|\mathrm{I}_{\nu_{j}}(X)\right|$. We stop when $\kappa_{m}$ is finite. For each $j<m$ let $\delta_{j}=\nu_{j+1}$ $-\nu_{j}$. Then the sequence $s_{j}=\left\langle\left|\mathrm{I}_{\nu_{j}+\delta}(X)\right|: \delta<\delta_{j}\right\rangle$ is in $S_{\kappa_{j}}$. Thus $\operatorname{CS}(X)=$ $s_{0} \frown s_{1} \frown \ldots \frown s_{m-1}$ provided $\kappa_{m}=0$ (i.e. $\mathrm{I}_{\nu_{m}}(X)=\emptyset$ ), and $\operatorname{CS}(X)=$ $s_{0} \frown s_{1} \frown \ldots \frown s_{m-1} \frown\left\langle\kappa_{m}\right\rangle$ when $0<\kappa_{m}<\omega$.

$(3) \Rightarrow(2)$. First we prove this implication for sequences $s$ of the form $s_{0} \frown s_{1} \frown \ldots \frown s_{m-1}$ by induction on $m$. If $s \in S_{\kappa_{0}}$ then the statement is just lemma 3.1

Assume now that $s=s_{0} \frown s_{1} \frown \ldots \frown s_{m-1}$, where $\kappa_{0}>\kappa_{1}>\cdots>\kappa_{m-1}$ and $s_{i} \in S_{\kappa_{i}}$ for $i<m$.

According to Lemma 3.1 there is a 0 -dimensional space $Y$ with cardinal sequence $s_{m-1}$. Using the inductive assumption we can also fix pairwise disjoint 0-dimensional topological spaces $X_{y, n}$ for $\langle y, n\rangle \in \mathrm{I}_{0}(Y) \times \omega$, each having the cardinal sequence $s^{\prime}=s_{0} \frown s_{1} \frown \ldots \frown s_{m-2}$. We then define the space $Z=\langle Z, \tau\rangle$ as follows. Let

$$
Z=Y \cup \bigcup\left\{X_{y, n}: y \in \mathrm{I}_{0}(Y), n<\omega\right\} .
$$

A set $U \subset Z$ is in $\tau$ iff

(i) $U \cap Y$ is open in $Y$,

(ii) $U \cap X_{y, n}$ is open in $X_{y, n}$ for each $\langle y, n\rangle \in \mathrm{I}_{0}(Y) \times \omega$,

(iii) if $y \in \mathrm{I}_{0}(Y) \cap U$ then there is $m<\omega$ such that $\bigcup\left\{X_{y, n}: m<n\right.$ $<\omega\} \subset U$.

If $U$ is a clopen subset of $Y$ and $n<\omega$ then it is easy to check that

$$
Z(U, n)=U \cup \bigcup\left\{X_{y, m}: y \in \mathrm{I}_{0}(Y) \cap U, n<m<\omega\right\}
$$

is clopen in $Z$. Hence

$$
\begin{aligned}
\mathcal{B}= & \{Z(U, n): U \subset Y \text { is clopen, } n<\omega\} \\
& \cup\left\{T: T \text { is a clopen subset of some } X_{y, n}\right\}
\end{aligned}
$$

is a clopen base of $Z$ and so $Z$ is 0 -dimensional.

Let $\delta^{\prime}=\operatorname{length}\left(s^{\prime}\right)$ and $\delta=$ length $(s)$.

Claim 3.2.1. $\mathrm{I}_{\alpha}(Z)=\bigcup\left\{\mathrm{I}_{\alpha}\left(X_{y, n}\right):\langle y, n\rangle \in \mathrm{I}_{0}(Y) \times \omega\right\}$ for $\alpha<\delta^{\prime}$. 
Proof. Since $X_{y, n}$ is an open subspace of $Z$ it follows that $\mathrm{I}_{\alpha}\left(X_{y, n}\right) \subset$ $\mathrm{I}_{\alpha}(Z)$. On the other hand,

$$
Y \subset{\overline{\bigcup\left\{\mathrm{I}_{\alpha}\left(X_{y, n}\right):\langle y, n\rangle \in \mathrm{I}_{0}(Y) \times \omega\right\}}}^{Z},
$$

hence $Y \cap \mathrm{I}_{\alpha}(Z)=\emptyset$. $\cdot 3.2 .1$

Since, by Claim 3.2.1,

$$
Z \backslash \bigcup_{\alpha<\delta^{\prime}} \mathrm{I}_{\alpha}(Z)=Y
$$

it follows that for $\delta^{\prime} \leq \alpha<\delta$ we have

$$
\mathrm{I}_{\alpha}(Z)=\mathrm{I}_{\alpha-\delta^{\prime}}(Y) .
$$

Thus $Z=\bigcup_{\alpha<\delta} \mathrm{I}_{\alpha}(Z)$, hence $Z$ is a scattered space of height $\delta$.

If $\alpha<\delta^{\prime}$ then, by Claim 3.2.1,

$$
\left|\mathrm{I}_{\alpha}(Z)\right|=\left|\mathrm{I}_{0}(y)\right| \cdot \omega \cdot s^{\prime}(\alpha)=\kappa_{m-1} \cdot \omega \cdot s^{\prime}(\alpha)=s^{\prime}(\alpha)=s(\alpha) .
$$

If $\delta^{\prime} \leq \alpha<\delta$ then, by $(*),\left|\mathrm{I}_{\alpha}(Z)\right|=\left|\mathrm{I}_{\alpha-\delta^{\prime}}(Y)\right|=s_{m-1}\left(\alpha \dot{-} \delta^{\prime}\right)=s(\alpha)$, and consequently $\operatorname{CS}(Z)=s$.

Thus we proved the statement for sequences of the form $s_{0} \frown \ldots \frown s_{m-1}$.

If $s=s_{0} \frown \ldots \frown s_{m-1} \frown\langle n\rangle$ then writing $s^{\prime}=s_{0} \frown \ldots \frown s_{m-1}$ we can first find pairwise disjoint 0-dimensional scattered spaces $X_{i, m},\langle i, m\rangle \in n \times \omega$, each having cardinal sequence $s^{\prime}$. Let

$$
Z=\left\{x_{i}: i<n\right\} \cup \bigcup\left\{X_{i, m}: i<n, m<\omega\right\} .
$$

Declare a set $U \subset Z$ open iff

(i) $U \cap X_{i, m}$ is open in $X_{i, m}$ for each $\langle i, m\rangle \in n \times \omega$,

(ii) if $x_{i} \in U$ then there is $n_{i}<\omega$ such that $\bigcup\left\{X_{i, m}: n_{i}<m<\omega\right\} \subset U$. Then $Z$ is 0 -dimensional, and

$$
\mathrm{I}_{\alpha}(Z)= \begin{cases}\bigcup\left\{\mathrm{I}_{\alpha}\left(X_{i, m}\right): i<n, m<\omega\right\} & \text { if } \alpha<\operatorname{length}\left(s^{\prime}\right), \\ \left\{x_{i}: i<n\right\} & \text { if } \alpha=\operatorname{length}\left(s^{\prime}\right) .\end{cases}
$$

Hence again $Z$ is a scattered space with $\operatorname{CS}(Z)=s$.

$(2) \Rightarrow(1)$ is trivial. -3.2

We leave it to the reader to verify that the sequences described in item (3) of Theorem 3.2 are exactly those mentioned at the beginning of the section with the additional obvious necessary condition that all but the last term of the sequence are infinite cardinals.

4. An alternative proof. We are grateful to the referee for pointing out to us the following alternative (and simpler) approach to obtain a proof of Theorem 2.1. This proof deduces 2.1 not from Theorem 2.2 but from the 
following weaker statement. We emphasize that the proof sketch given below is due to the referee.

THEOREM 4.1. In $V$, set $\kappa=\left(2^{\omega}\right)^{+}$. Let $V[G]$ be formed by adding any number of Cohen reals to $V$. Then in $V[G]$, the following holds: Suppose we are given:

(1) a separable zero-dimensional $T_{3}$ space $X$,

(2) pairwise disjoint countable $E_{\alpha}$ for $\alpha<\kappa$ such that $\bar{E}_{\alpha} \supset E_{\beta}$ for $\alpha<\beta<\kappa$.

Then we can find a sequence $\left\{K_{n}: n \in \omega\right\}$ of clopen sets which is independent; that is, if we let $K_{n}^{\mu}$ be $K_{n}$ if $\mu=0$ and $X \backslash K_{n}$ if $\mu=1$, then $\bigcap_{i<n} K_{n}^{s(n)} \neq \emptyset$ for each $n<\omega$ and $s \in 2^{n}$.

Proof. To prove this, first work in $V[G]$. Assume that $\omega \subset X$ and $\omega$ is dense in $X$. For each $\alpha<\kappa$, let $B_{\alpha} \subset \mathcal{P}(X)$ be a countable subalgebra of the clopen sets such that $B_{\alpha}$ separates the points of $E_{\alpha}$. Let $C_{\alpha}=\{K \cap \omega$ : $\left.K \in B_{\alpha}\right\}$. Then $C_{\alpha}$ is a countable subalgebra of $\mathcal{P}(\omega)$. Observe that the map $K \mapsto K \cap \omega$ is an isomorphism from $B_{\alpha}$ onto $C_{\alpha}$; its inverse is the map $H \mapsto \bar{H}$.

Still in $V[G]$, let $T$ be the tree of height $\omega$ whose nodes at level $n$ are pairs $(\vec{\alpha}, \vec{H})$, where $\vec{\alpha}=\left\langle\alpha_{i}: i<n\right\rangle \in \kappa^{n}$ is a sequence of distinct ordinals and $\vec{H}=\left\langle H_{i}: i<n\right\rangle \in(\mathcal{P}(\omega))^{n}$ is an independent sequence, with each $H_{i} \in C_{\alpha_{i}}$. Use $<$ for the usual tree order, with the root $(\emptyset, \emptyset)$ at the top. Assume that there is no independent $\omega$-sequence, as claimed in the theorem. Then $T$ must be well-founded, and hence has an ordinal-valued rank function, $\varrho$, defined by $\varrho(x)=\sup \{\varrho(y)+1: y \in T \wedge y<x\}$.

Still in $V[G]$, we note that since $\kappa \geq \omega_{2}, T$ must have nodes of uncountable rank. To prove this, call a node $x=(\vec{\alpha}, \vec{H})$ special if $\alpha_{0}>$ $\alpha_{1}>\cdots>\alpha_{n-1}$ and each $\bigcap_{i<n} K_{i}^{s(i)}$ (for $s \in 2^{n}$ ) meets $E_{\alpha_{n-1}}$, where $K_{i}=\bar{H}_{i}$. We prove that $\varrho(x) \geq \alpha_{n-1}$ for such special nodes $x$. The proof proceeds by induction on $\alpha_{n-1}$; it is sufficient to prove that for each $\beta<\alpha_{n-1}: x$ has an extension of the form $y=\left(\overrightarrow{\alpha^{\prime}}, \overrightarrow{H^{\prime}}\right)$, where $y$ is special, $\overrightarrow{\alpha^{\prime}}=\left\langle\alpha_{0}, \alpha_{1}, \ldots, \alpha_{n-1}, \alpha_{n}\right\rangle$, with $\alpha_{n}=\beta$, and $\overrightarrow{H^{\prime}}$ extends $\vec{H}$; so, we need to define $H_{n}$. Since each clopen set $\bigcap_{i<n} K_{i}^{s(i)}$ meets $E_{\alpha_{n-1}}$, and $\bar{E}_{\beta} \supset E_{\alpha_{n-1}}$, each $\bigcap_{i<n} K_{i}^{s(i)}$ meets $E_{\beta}$ in an infinite set. Since $B_{\beta}$ separates points of $E_{\beta}$, we can choose $K_{n}$ so that it and its complement meet all $2^{n}$ sets $E_{\beta} \cap \bigcap_{i<n} K_{i}^{s(i)}$. Then let $H_{n}=K_{n} \cap \omega$.

Now, in $V$, we have, for each $\alpha<\kappa$, a name $\dot{C}_{\alpha}$ which is forced to name a countable subalgebra of $\mathcal{P}(\omega)$. Since $\kappa=\left(2^{\omega}\right)^{+}$, we may apply a $\Delta$-system and thinning-out argument and assume, without loss of generality, that the names $\dot{C}_{\alpha}$ are disjointly supported and isomorphic; this means that given 
any permutation $\pi$ of $\kappa$, there is an automorphism $\sigma$ of the forcing order $\mathbb{P}$ such that each $\sigma\left(\dot{C}_{\alpha}\right)=\dot{C}_{\pi(\alpha)}$. We do this thinning-out before we define the tree $\dot{T}$. We also have a name $\dot{\varrho}$ for the rank function. But now, using the automorphisms, it is easily proved that $\varrho$ is forced to take on only countable values, which is a contradiction. To see this: whenever $\vec{\alpha} \in \kappa^{<\omega}$ (in $V$ ) is a sequence of distinct ordinals, let

$$
R_{\vec{\alpha}}=\{\xi: \exists p \in \mathbb{P}[p \Vdash(\exists \vec{H}[(\vec{\alpha}, \vec{H}) \in \dot{T} \wedge \dot{\varrho}(\vec{\alpha}, \vec{H})=\xi])]\} .
$$

Then each $R_{\vec{\alpha}}$ is countable (by the ccc) and $R_{\vec{\alpha}}$ only depends on the length of $\vec{\alpha}$ (by the automorphisms). It follows that the union of all the $R_{\vec{\alpha}}$ is countable. Since this union must also be an initial segment of the ordinals, it is forced that $\dot{\varrho}$ only takes countable values.

\section{References}

[1] J. E. Baumgartner and S. Shelah, Remarks on superatomic Boolean algebras, Ann. Pure Appl. Logic 33 (1987), 109-129.

[2] J. Brendle and S. Fuchino, Coloring ordinals by reals, preprint.

[3] I. Juhász and K. Kunen, The power set of $\omega$, elementary submodels and weakenings of CH, Fund. Math. 170 (2001), 257-265.

[4] I. Juhász, L. Soukup, and Z. Szentmiklóssy, Combinatorial principles from adding Cohen reals, in: Logic Colloquium '95 (Haifa), J. A. Makowsky (ed.), Lecture Notes in Logic 11, Springer, 1998, 79-103.

[5] I. Juhász and W. Weiss, On thin-tall scattered spaces, Colloq. Math. 40 (1978), 63-68.

[6] W. Just, Two consistency results concerning thin-tall Boolean algebras, Algebra Universalis 20 (1985), 135-142.

[7] K. Kunen, Set Theory, North-Holland, New York, 1980.

[8] J. C. Martínez, A consistency result on thin-very tall Boolean algebras, Israel J. Math. 123 (2001), 273-284.

Alfréd Rényi Institute of Mathematics

Reáltanoda u. 13-15

H-1053 Budapest, Hungary

E-mail: juhasz@renyi.hu soukup@renyi.hu

Department of Analysis

Eötvös Loránd University

Pázmány Péter sétány 1/c

H-1117 Budapest, Hungary

E-mail: zoli@renyi.hu
Institute of Mathematics The Hebrew University of Jerusalem 91904 Jerusalem, Israel E-mail: shelah@math.huji.ac.il

Received 21 July 2003; in revised form 2 March 2004 